\title{
1 Genome assembly of the maize inbred line A188 provides a new reference genome for functional genomics
}

3 Fei Ge ${ }^{1 \#}$, Jingtao $\mathrm{Qu}^{1 \#}$, Peng Liu ${ }^{1 \#}$, Lang Pan ${ }^{1 \#}$, Chaoying Zou, Guangsheng Yuan ${ }^{1}$, Cong Yang ${ }^{1}$,

4 Shibin $\mathrm{Gao}^{1}$, Guangtang $\operatorname{Pan}^{1}$, Jianwei Huang ${ }^{2}$, Langlang Ma ${ }^{1 *}$, Yaou Shen ${ }^{1 *}$

$5 \quad{ }^{1}$ Key Laboratory of Biology and Genetic Improvement of Maize in Southwest Region, Maize Research Institute,

6 Sichuan Agricultural University, Chengdu, 611130, China

$7 \quad{ }^{2}$ Berry Genomics Corporation, Beijing, 100015, China

$8 \quad$ \# $T$ These authors contributed equally to this work

9 *Corresponding authors:

10 Langlang Ma, email: sxyljxml@163.com

11 Yaou Shen, email: shenyaou@ sicau.edu.cn

12 Highlight

13 Our manuscript presents a high-quality reference genome of the inbred line A188, and

14 provides new insights into candidate genes underlying maize embryonic callus

15 induction and other maize agronomic traits.

\section{Abstract}

17 Heretofore, little is known about the mechanism underlying the genotype-dependence

18 of embryonic callus (EC) induction, which has severely inhibited the development of

19 maize genetic engineering. Here, we report the genome sequence and annotation of a

20 maize inbred line with high EC induction ratio, A188, which is assembled from

21 single-molecule sequencing and optical genome mapping. We assembled a 2,210 Mb

22 genome with a scaffold N50 size of 11.61 million bases (Mb), compared to those of

$239.73 \mathrm{Mb}$ for B73 and 10.2 Mb for Mo17. Comparative analysis revealed that $~ 30 \%$ of

24 the predicted A188 genes had large structural variations to B73, Mo17 and W22 
1 genomes, which caused considerable protein divergence and might lead to phenotypic

2 variations between the four inbred lines. Combining our new A188 genome,

3 previously reported QTLs and RNA sequencing data, we reveal 8 large structural

4 variation genes and 4 differentially expressed genes playing potential roles in EC

5 induction.

\section{$6 \quad$ Keywords}

7 Maize, Embryonic callus, A188, Single-molecule sequencing, RNA sequencing,

8 Genome assembly

\section{Introduction}

10 Genetic transformation has been an effective approach for elucidating gene functions

11 in plants. Maize (Zea mays L.) genetic transformation is highly relied on the

12 utilization of embryonic callus (EC) induced from immature embryos. However, only

13 a few lines possess the ability to efficiently form embryonic callus, including several

14 inbred lines as A188, B104, H99, C01 and the combination Hi-II (A×B) etc.

15 (Armstrong et al., 1992; Bronsema et al., 1997; Krakowsky et al., 2006; Salvo et al.,

16 2018). Since the plant regeneration from maize tissue culture was firstly reported in

171975 (Green and Phillips, 1975), little is known about how the maize EC was induced

18 from the immature embryos even though great efforts have been made by generation

19 to generation of researchers (Armstrong et al., 1992; Krakowsky et al., 2006; Pan et

20 al., 2006; Salvo et al., 2018). To date, only several QTLs were identified involved in

21 controlling callus induction or plant regeneration.

22 Using an $\mathrm{F}_{2}$ population derived from two inbred lines with divergent $\mathrm{EC}$ 
1 induction rate, Pan et al. mapped 5 QTLs for tissue culture response on chromosome

$2 \quad 1,3,7$ and 8, respectively (Pan et al., 2006). Similarly, several type I callus formation

3 related QTLs were identified using an $\mathrm{F}_{6}$ RIL population constructed from $\mathrm{H} 99$ and

4 Mo17 (Krakowsky et al., 2006). More QTLs responsible for plantlets and

5 transformation were identified using a segregation population constructed from

6 FBLLxHi-II (Lowe et al., 2006). Armstrong et al. used a low frequency of EC

7 initiation line B73 to backcross a high tissue culture response line A188, generating

8 the $\mathrm{BC}_{6} \mathrm{~S}_{4}$ lines with high frequency of EC initiation (Armstrong et al., 1992). Five

9 introgressed A188 segments were identified correlating with EC formation

10 (Armstrong et al., 1992), and 4 markers located in or near the introgressed A188

11 segments were found involving EC formation using an $\mathrm{F}_{2}$ population of A188 $\times$ Mo17

12 (Armstrong et al., 1992). Similarly, a B73 Near isogenic line WCIC2 (Donor parent:

13 A188) with high frequency of EC initiation was used to genetically fine-map the

14 QTLs for EC response, finally, a QTL located within a $3.06 \mathrm{Mb}$ region on

15 chromosome 3 was identified to control EC formation and regeneration (Salvo et al.,

16 2018). Through reverse genetics, two genes, ZmWUS2 and ZmBBM, were proved to

17 regulate maize EC formation and regeneration. Overexpression of the two genes

18 resulted in the improved frequencies of EC induction and transformation in both

19 immature embryos and mature explants of the inbred lines with low tissue culture

20 response (Lowe et al., 2016). However, few of genes responsible for tissue culture

21 response were cloned in maize.

22 Maize shows remarkable genomic diversity among various inbred lines (Buckler 
1 et al., 2006; Lai et al., 2010; Schnable et al., 2009; Springer et al., 2009). The

2 temperate line A188 (Gacheri et al., 2015), with high frequency of EC initiation

3 (Armstrong et al., 1992; Salvo et al., 2018), is a desirable material to study the

4 molecular mechanism underlying EC formation and regeneration. Due to the

5 difference between A188 genome and B73 reference genome, these identified QTLs

6 for embryo culture response have not been cloned so far, limiting their applications in

7 improving EC formation capability. In addition, A188 shows considerable phenotypic

8 variations from other inbred lines, such as plant height (Peiffer et al., 2014), ear

9 height, days to tassel (Peiffer et al., 2014), days to silk (Peiffer et al., 2014), oil

10 concentration (Cook et al., 2011), protein concentration (Cook et al., 2011), starch

11 concentration (Cook et al., 2011) etc. (Table 1). Collectively, the assembly of a

12 high-quality A188 reference genome is helpful to reveal the molecular mechanism

13 underlying EC induction and other agronomic traits. Here we combine

14 single-molecule sequencing and BioNano optical-mapping technologies to produce a

15 de novo assembly of the A188 genome and provide these research communities with

16 an excellent resource.

\section{Methods}

18 Phenotypic evaluations of maize inbred line A188, B73, Mo17 and W22. The

19 maize (Zea Mays L.) inbred lines A188, B73, Mo17 and W22, provided by Maize

20 Research Institute of Sichuan Agricultural University, were grown in Chengdu

21 (Sichuan province, China, N3067', E104 ${ }^{\circ}$ 06') in 2018. All of the lines were planted

22 in a randomized complete block design with three replicates and two rows per line. A 
1 total of 14 plants were contained per row with the row length of $3 \mathrm{~m}$ and the row

2 ledge of $0.75 \mathrm{~m}$. These materials were managed according to the standard cultivation

3 practices. At 10 days after pollination (DAP), the plant height and TBN were

4 measured as described previously (Brown et al., 2011). The day duration from

5 seeding to half of the plants tasseled, pollinated and silked was recorded as days to

6 tassel, days to pollination and days to silk, respectively. The ear numbers were

7 counted at 30 DAPs. Three mature seeds of each inbred line were crushed and

8 subjected to the measurement of the protein concentration using the RAPID $\mathrm{N}$

9 exceed® (Elementar, Straße 163505 Langenselbold, Germany) according to the

10 manufacturer's instructions.

11 Maize inbred lines A188, B73, Mo17 and W22 were planted in a greenhouse

$12\left(14 / 10 \mathrm{~h}\right.$ light/dark, at $28^{\circ} \mathrm{C}$ and $70 \%$ relative humidity). Twelve days after

13 self-pollination, 108 immature embryos (with 1.2-1.5 $\mathrm{mm}$ in length) from each line

14 were collected, and evenly distributed among three Petri dishes containing the

15 modified N6 medium (Frame et al., 2002) with scutellum upward to induce EC, with

16 three replicates. After aseptic incubation for 21 days in darkness at $28^{\circ} \mathrm{C}$, we

17 investigated the EC induction ratio which was represented by (number of the

18 immature embryos successfully inducing EC/ number of inoculated immature

19 embryos) $\times 100 \%$.

20 Genomic DNA and total RNA isolation. The plants of A188 were grown in a

21 greenhouse at $28 \square$ in a dark condition for $14 \mathrm{~d}$. The yellow leaves of A188 seedlings

22 were isolated and frozen immediately in liquid nitrogen for extracting genomic DNA, 
1 which was subsequently used for constructing libraries for PacBio sequencing and

2 BioNano optical maps. To assist gene annotation and transcriptome analysis,

3 transcriptome of five tissues were performed single-molecule long-read sequencing.

$4 \quad$ Total RNA was extracted from five tissues (12-d seedlings, tassel, silk, pericarp

5 and 20-DAP seeds) with TRIzol reagent (Invitrogen, Carlsbad, CA) according to the

6 manufacturer's instructions. For each sample, we generated three independent

7 biological replicates. The same amount of RNAs $(1 \mu \mathrm{g})$ for each replicate of each

$8 \quad$ tissue were pooled and stored at $-80 \square$.

9 PacBio library construction and sequencing. Libraries for SMRT PacBio genome

10 sequencing were constructed as previously reported (Pendleton et al., 2015). Briefly,

$1120 \mu \mathrm{g}$ of high-quality genomic DNA was sheared, and the $20 \mathrm{~kb}$ targeted size

12 fragments were selected for ligation with SMRT adapter, followed by purification and

13 size selection with Agilent 2100. The obtained PCR-free SMRTbell libraries were

14 sequenced on the PacBio Sequel platform (Pacific Biosciences).

15 One microgram of enriched polyA RNA was reversely transcribed into cDNA by

16 using the Clontech SMARTER cDNA synthesis kit, which was subjected to size

17 selection using the BluePippin system. Size fractions eluted from the run were

18 re-amplified to generate 2 libraries $(0-1 \mathrm{~kb}$ and 1-10 kb). Then $2 \mu \mathrm{g}$ cDNA of each

19 library were subjected to Iso-Seq SMRTBell library construction according to the

20 protocol reported on the website (https://pacbio.secure.force.com/SamplePrep). The

21 SMRTBell libraries were then subjected to single-molecule sequencing on the PacBio

22 Sequel platform (Pacific Biosciences). 
1 Optical library construction and sequencing. The professional kit was used to

2 extract the High Molecular Weight (HMW) genome by agarose-embedded cells

3 followed by protein digestion. The HMW genome of the quality check-through was

4 specifically recognized by the $B s p Q$ I enzyme to identify the site to be labeled, and

5 the fluorescent group is added to the double-stranded DNA molecule by means of

6 modification and labeling, thereby ensuring the stability of the double strand and

7 increasing sequence tag density at the same time. Finally, DNA molecules with

8 fluorescent tags are stained to complete genome-specific tagging.

9 The marked library was loaded on the Irys chip for scanning and photographing.

10 During the whole process, images were continuously converted into map data. After

11 the real-time statistics and quality of Access reached the standard, the instrument was

12 stopped and the data was transferred to bioinformatics analysis.

13 De novo assembly of PacBio SMRT reads. After removing the short polymerase

14 reads, low quality polymerase reads and self-connected adaptor sequences,

$1527,248,178$ subreads (approximately 224G) were used for contig assembly with

16 Falcon (Pendleton et al., 2015). Firstly, all of the subreads were pairwise compared to

17 correct the error sequence with the parameters '--length_cutoff 12000

18 --length_cutoff_pr 14000', followed by preliminary assembly with parameters:

19 --min_idt 0.70 --min_cov 2 --max_n_read 200, and further error correction. The

20 overlap graphs were constructed with parameters '--max_diff 100 --max_cov 100

21 --min_cov 2', and then the contigs were assembled based on it. The Blasr (Chaisson

22 and Tesler, 2012) was employed to map all the subreads back to the contigs with the 
1 parameters '--bestn 1 --maxScore -1000 --hitPolicy randombest'. We further

2 performed assembly error corrections using Arrow

3 (https://github.com/PacificBiosciences/GenomicConsensus/) with default parameters.

4 Construction of BioNano optical maps. High-molecular-weight DNA was digested

5 by the endonuclease BspQI and then labeled with IrysPrep Labeling mix and Taq

6 polymerase according to standard BioNano protocols. The BioNano raw data was

7 filtered using Bionano Access (version 1.0.3), generating high quality data. Then the

8 BioNano Irys system was subsequently used to automatically image the labeled DNA.

9 IrysSolve (https://bionanogenomics.com/support/software-downloads/) was used to

10 de novo assemble the BioNano bnx files into genome maps. The RefAligner

11 (https://bionanogenomics.com/support/software-downloads/) was used for molecule

12 Pairwise comparison to identify overlaps, followed by construction of consensus

13 maps. We recursively refined and extended the consensus maps by mapping all

14 molecules back to the consensus maps.

15 Hybrid assembly of PacBio contigs and BioNano optical maps. The

16 PacBio-assembled contigs and BioNano-assembled genome maps were subjected to

17 hybrid assembly by using the 'HybridScaffold' module of the IrysSolve as described

18 previously (Sun et al., 2018). Briefly, the PacBio genome maps were aligned to an in

19 silico BspQI-digested cmap. The BioNano genome maps were then aligned to the

20 PacBio genome maps with RefAligner, followed by identifying and resolving the

21 conflict points. After resolving the conflict points, Bionano genome maps and PacBio

22 genome maps were merged to generate hybrid scaffold. The PacBio genome maps 
1 were mapped to hybrid scaffolds again to identify overlaps. If the overlap between

2 PacBio contigs and hybrid scaffold was longer than $1 \mathrm{~kb}$ and identity $\geq 95 \%$, these

3 two sequences were merged. Based on the alignment information, the super-scaffolds

4 were built.

5 Construction of pseudomolecules. The A188 scaffolds were mapped to

6 B73_RefGen_v4 genome using Bwa (version bwa-0.7.15,

7 http://bio-bwa.sourceforge.net/bwa.shtml). The mapping rate of each scaffold mapped

8 to each chromosome of B73 were calculated. The scaffolds with the highest mapping

9 rate were kept to determine mapping to which B73 chromosome. The mapping file

10 was further filtered according to the one-to-one correspondence, and the comparison

11 noise was excluded. The remaining mapping results were submitted to combining

12 coordinate to determine the alignment of scaffold on the B73 chromosome. Reverse

13 alignments of the scaffolds were also performed based on the filtered mapping result

14 file, and finally the scaffolds were anchored to the B73 reference genome according

15 to the filtered position alignment information.

16 Assembly evaluation. BUSCO (Benchmarking Universal Single-Copy Orthologs:

17 http://busco.ezlab.org/) combined with tblastn, augustus, and hmmer softwares were

18 used to evaluate the genome-assembly completeness. 'Embryophyta_odb9' that

19 contained 1,440 single-copy orthologous genes was used as a searching dataset and

20 was employed to assess the assembly completeness of the A188 genome.

21 Repetitive elements prediction.

22 TRF v4.07b (http://tandem.bu.edu/trf/trf407b.linux64.download.html) was used to 
1 predict tandem repeat. LTR Finder (Xu et al., 2010), RepeatScout (v1.0.5,

2 http://www.repeatmasker.org), and PILER (v1.0, http://www.drive5.com/piler) were

3 used to predict LTR element, LINE, SINE, and transposable DNA, respectively. First

4 of all, the low complexity and low copy results of RepeatScout and PILER were

5 removed. The predicted repetitive element sequences longer than $100 \mathrm{bp}$ and the gap

6 length $\leq 5 \%$ were kept and further mapped to protein sequences in SwissProt

7 (ftp://ftp.ebi.ac.uk/pub/databases/uniprot/knowledgebase/uniprot_sprot.fasta.gz), the

8 sequence alignments with non-transposable element protein sequences with evalue $\leq$

$91 \mathrm{e}^{-4}$, identity $\geq 30$, coverage $\geq 30 \%$, and length $\geq 90$ bp were removed. Then the

10 remained sequences were aligned to Rfam 11.0 database

11 (ftp://ftp.ebi.ac.uk/pub/databases/Rfam) using BLASTN to remove ncRNA, and the

12 predicted repetitive elements with evalue $\leq 1 \mathrm{e}^{-10}$, identity $\geq 80$, and coverage $\geq 50 \%$

13 were removed. Moreover, the remained repetitive elements were aligned to RepBase

14 and TE protein database using WU-BLAST, and were classified using

15 RepeatClassifier, with the known simple repeat, satellite, and ncRNA sequences

16 removed. The remained repetitive elements were compared to each other using

17 BLASTN, the sequences with evalue $\leq 1 \mathrm{e}^{-10}$, identity $\geq 80$, coverage $\geq 80 \%$, and

18 mapping length $\geq 80$ bp were removed. Finally, the interspersed repeats were

19 generated by masking predicted repetitive elements, known repetitive elements

20 (RepBase), and protein repeat sequence (TE protein database) using RepeatMasker,

21 RepeatMasker, and RepeatProteinMask, respectively.

\section{Gene annotation}


1 MAKER2 (http://www.yandell-lab.org/software/maker.html) (Cantarel et al., 2008)

2 was used to annotate genes in the A188 genome with the strategy as described

3 previously (Sun et al., 2018). First, for protein-homology-based prediction, we

4 downloaded the proteins of B73 reference genome, Mo17 reference genome, and

5 W22 reference genome from gramene (http://gramene.org/) (Tello-Ruiz et al., 2015)

6 as input of MAKER2. The A188 transcripts assembled from five different tissues

7 based on single-molecule long-read sequencing in this study, B73 full-length

8 transcripts from Iso-seq (Wang et al., 2016), and Mo17 transcripts (Sun et al., 2018)

9 were used for gene transcript prediction. Second, the generated gene models were

10 submitted to Augustus (Keller et al., 2011), SNAP

11 (http://snap.stanford.edu/snap/download.html), GeneMark-ESSuite (version 4.32

12 http://topaz.gatech.edu/GeneMark/license_download.cgi), and Glimmerhmm

13 (http://ccb.jhu.edu/software/glimmerhmm/) ab initio prediction softwares to further de

14 novo predict gene models. Then, we further filtered the preliminary prediction gene

15 set according to AED scores generated in MAKER software and the high confidence

16 gene models generated finally.

\section{Identification of PAV sequences.}

18 To identify presence/absence-variation sequences (PAV, length longer than 500bp),

19 we used a sliding-window method as reported previously (Sun et al., 2018). To

20 identify A188-specific PAV sequences to B73, the A188 genome was divided into

$21500 \mathrm{bp}$ windows with a step size of $100 \mathrm{bp}$. Then all of the $500 \mathrm{bp}$ windows were

22 aligned to B73 genome with BWA mem (Li, 2013) (http://bio- bwa.sourceforge.net/) 
1 with options '-w 500 -M'. The A188-sepecific PAV sequences are the sequences that

2 cannot be aligned to the B73 genome or the primary alignment coverage less than $25 \%$

3 (Sun et al., 2018). Two overlapped PAV windows were merged. The same method

4 was used to identify A188-specific PAV sequences to Mo17 and to W22,

5 B73-specific PAV sequences to A188, Mo17-specific PAV sequences to A188, and

6 W22-specific PAV sequences to A188. The PAV sequences within $100 \mathrm{~kb}$ of the

7 physical coordinates were further merged. The merged region had more than $10 \%$

8 PAV sequences were defined as a PAV cluster. Finally, all of the PAVs were

9 anchored back to corresponding genome. We used the same method (Sun et al., 2018)

10 to identify A188-specific genes to B73, Mo17 and W22, respectively. In brief, the

11 genes with more than $75 \%$ of the CDS regions falling in PAV sequences were defined

12 as PAV genes.

\section{Comparative genomic analysis among A188, B73, Mo17 and W22.}

14 The Mummer software (Kurtz et al., 2004) was used to perform comparative genomic

15 analysis between A188 and B73 genomes. Each A188 pseudo-chromosome sequence

16 was mapped to the corresponding B73 chromosome using mummer with the

17 parameters "nucmer -g 1000 -c 90 -1 40". The mapping results were submitted to

18 delta-filter to perform noise filtration with parameters "-r -q". The show-coords was

19 used for conversion of aligned physical coordinates with parameters "-rcloTH". SNPs

20 and small InDels (<100 bp) were identified using show-snps with "-ClrTH".

21 Show-diff was employed to obtain Inversions with the default parameters. Finally, we

22 further filtered the alignments with aligned physical positions in one genome that 
1 were located more than $10 \mathrm{Mb}$ away in another genome. The comparative genomic

2 analysis between A188 and Mo17, A188 and W22 genomes were processed with the

3 same method.

4 Results

5 Remarkable phenotypic difference between A188 and the other 6 assembled lines

7 As a public inbred line, A188 has an outstanding response to the tissue culture,

8 generating an approximate $100 \%$ efficiency in forming EC from immature embryos

9 (Hodges et al., 1986; Ishida et al., 1996). However, previous studies demonstrated

10 that B73 and Mo17 both have a very low frequency of inducing EC under standard

11 conditions (Frame et al., 2006; Salvo et al., 2018). We also compared the phenotype

12 of EC formation ratio among A188, B73, Mo17 and W22, indicating the highest EC

13 induction ratio of A188 and the low EC induction ratios of the other three lines (Table

14 1). Moreover, the other agronomic traits show significant difference between A188

15 and the other lines including plant height, tassel branch numbers, ear numbers, protein

16 concentration, days to tassel, days to pollination, and days to silk (Table 1 and Fig. 1).

17 Our findings were in agreement with the previous studies about the phenotypic

18 performances of A188 (Cook et al., 2011; Peiffer et al., 2014), implicating that

19 A188/B73, A188/Mo17 and A188/W22 are therefore the ideal pairs of maize lines in

20 genetic and molecular studies of these traits.

\section{Genome sequencing and de novo assembly}

22 By combining with optical genome mapping with the BioNano Genomics Irys System, 
1 PacBio Sequel platform was used to sequence and de novo assemble of the A188

2 genome. Firstly, over 104-fold coverage of sequence data (224.03 Gb in total)

3 generated from PacBio Sequel technology was used to perform initial assembly,

4 resulting in a $2127.72 \mathrm{Mb}$ assembly with a contig N50 size of $1.06 \mathrm{Mb}$ and the

5 longest contig of $4.97 \mathrm{Mb}$ (Table 2, Tables S1 and S2). We then used 631.48-Gb

6 BioNano molecule (287-fold-coverage BioNano optical map) to scaffold the

7 assembled contigs and generated the final assembly which contains 4,469 scaffolds

8 with a scaffold $\mathrm{N} 50$ size of $11.61 \mathrm{Mb}$ and the longest length of $47.84 \mathrm{Mb}$ (Table 2 and

9 Tables S1 and S2). The total size of the final assembly was 2,207.74 Mb, similar to

10 those of the B73 (2,106 Mb) (Jiao et al., 2017), Mo17 (2,183 Mb) (Sun et al., 2018)

11 and SK (2,094 Mb) (Yang et al., 2019) genomes (Table 2 and Table S2). Bwa-0.7.15

12 was then used to anchor the A188 scaffolds to ten pseudo-chromosomes based on the

13 B73 RefGen v4 genome according to the filtered position alignment information

14 (Methods). Finally, 295 scaffolds were anchored and oriented onto ten chromosomes

$15(2,084.35 \mathrm{Mb}, 94.30 \%$ of the final genome assembly) and 3704 scaffolds failed to be

16 mapped to chromosomes (5.70\% of the final genome assembly) (Table S3). The final

17 A188 assembly had 2,480 gaps (89.56 Mb in length), compared with 2,522 gaps in

18 B73 and 238 gaps in SK genome (Yang et al., 2019). BUSCO (Simão et al., 2015)

19 was used to evaluate the A188 genome assembly quality. Finally, 95.3\% of complete

20 single-copy BUSCOs could be aligned to the A188 final assembly, similar to those

21 for the B73 (Jiao et al., 2017), Mo17 (Sun et al., 2018), W22 (Springer et al., 2018)

22 and SK (Yang et al., 2019) genomes, indicating the near completeness of our 
1 assembly (Table S4).

\section{Genome annotation}

3 A modified approach based on the annotation pipeline (Sun et al., 2018) was

4 employed to analyze the transposable-element content of our A188 assembly. Finally,

5 approximately $80.70 \%$ of the A188 genome sequence were identified as

6 transposable-element sequences, including retrotransposons (71.93\%), DNA

7 transposons (5.91\%), and unclassified elements (2.49\%) (Table S5), which was lower

8 than those in B73 (Jiao et al., 2017), Mo17 (Sun et al., 2018), W22 (Springer et al.,

9 2018), SK (Yang et al., 2019) and K0326Y (Li et al., 2020) genomes. For

10 retrotransposons, the families of Copia and Gypsy represented $24.01 \%$ and $46.92 \%$ of

11 the A188 genome, respectively (Table S5). For DNA transposons, the family of $h A T$

12 was much lower than those in the B73 (Jiao et al., 2017) and Mo17 (Sun et al., 2018)

13 genomes.

14 To annotate the A188 genes, we integrated two technologies,

15 protein-homology-based prediction and isoform sequencing of five different A188

16 tissues, and combined the reported B73 full-length transcripts (Wang et al., 2016) and

17 Mo17 transcripts (Sun et al., 2018). In total, 44,653 high-confidence protein-coding

18 gene models with 66,359 transcripts were predicted (Table S6). Among them, 10,965

19 (24.56\% of the predicted genes) and 16,243 (36.38\% of the predicted genes) genes

20 were supported by ISO-seq with CDS coverage $>90 \%$ and $>50 \%$, respectively (Table

21 S6). In total, $41,715(93.42 \%)$ of the predicted A188 genes were mapped to ten

22 pseudo-chromosomes (Table S3). In addition, 93.52\% $(62,058)$ of these transcripts 
1 were functionally annotated and deposited in the public databases (Fig. S1).

\section{Genome structural variations between A188, B73, Mo17 and W22}

3 To better understand the genome difference, we individually aligned the

4 pseudo-chromosomes of B73, Mo17 and W22 to those of A188. In total, $62.50 \%$

$5 \quad(1,316.38 \mathrm{Mb}), 63.10 \%(1,327.82 \mathrm{Mb})$ and $62.59 \%(1,327.48 \mathrm{Mb})$ of the B73, Mo17

6 and W22 genome sequences matched in one-to-one syntenic blocks with $63.16 \%$

$7 \quad(1,316.45 \mathrm{Mb}), 63.71 \%(1,328.03 \mathrm{Mb})$, and $63.69 \%(1,327.43 \mathrm{Mb})$ of the $\mathrm{A} 188$

8 genome sequence, respectively (Fig. 2, Fig. S2, and Table S7).

A total of 9,865,320 SNPs, 634,693 insertions and 654,322 deletions were

10 identified between A188 and B73, with an average of 4.73 SNPs, 0.30 insertions and

110.31 deletions per kilobase (Fig. 2, Fig. S3, and Table S7). We also identified

$129,490,058$ SNPs, 743,829 insertions and 654,841 deletions between A188 and Mo17,

13 and 9,614,783 SNPs, 600,755 insertions and 628,504 deletions between A188 and

14 W22 (Fig. S3, Table S7). Less than 2.5\% of these variations in A188 are allocated in

15 CDS regions, and the remainders are annotated as intergenic variations (Table 3 and

16 Table S8). Interestingly, a genome-wide comparison showed that InDels of $3 \mathrm{~N} \pm 1 \mathrm{bp}$

17 in $\mathrm{CDS}$ region were more abundant than $3 \mathrm{~N}$ bp in gene coding regions (Table 3 ),

18 between A188 and any one of lines B73, Mo17 and W22. We then focused on

19 identifying presence/absence-variation sequences (PAV) longer than $500 \mathrm{bp}$ in the

20 A188 genome. By comparing the A188 and B73 genomes, we identified 27,240

21 A188-specific genomic segments (16.92 $\mathrm{Mb}$ in total) and 28,558 B73-specific

22 genomic segments $(17.76 \mathrm{Mb}$ in total). Most of these PAV segments were shorter 
1 than $3 \mathrm{~kb}$, only 1 and 2 PAV segments were identified longer than $3 \mathrm{~kb}$ in A188 and

2 B73, respectively (Fig. S4). Similarly, by comparing the A188 and Mo17 genomes,

3 we identified 26,983 A188-specific genomic segments (16.76 Mb in total), and

4 28,030 Mo17-specific genomic segments (17.44 Mb in total). Most of the PAV

5 segments were shorter than $3 \mathrm{~kb}$, only 1 and 3 PAV segments were identified longer

6 than $3 \mathrm{~kb}$ in A188 and Mo17, respectively (Fig. S4). The comparison of the A188 and

7 W22 genomes identified 31,536 A188-specific genomic segments (19.42 Mb in total),

8 and 29,192 W22-specific genomic segments (17.98 Mb in total), with 1 and 4 PAV

9 segments longer than $3 \mathrm{~kb}$ in A188 and W22, respectively (Fig. S4). According to the

10 criterion that a gene with $\geq 75 \%$ of coding sequences covered by a PAV sequence can

11 be identified as a PAV gene (Sun et al., 2018), we identified 100 A188-specific and

12104 B73-specific PAV genes, by comparison of A188 and B73 genomes. Similarly,

13116 A188-specific and 146 Mo17-specific PAV genes were found by comparing

14 A188 and Mo17, and 112 A188-specific and 116 W22-specific PAV genes were

15 identified between A188 and W22 (Table 3 and Table S9). Combined these findings

16 illustrated that the A188 genome showed huge variations from B73, Mo17 and W22

17 genomes. However, only 9 A188-specific PAV genes were simultaneously identified

18 in comparison with the other three inbred lines, thus illustrating that most of the

19 A188-specific PAV genes have already existed in other lines (Table S9).

\section{Gene structural variations}

21 A total of $20,557,21,007$ and 20,713 genes displayed no variations in the CDS

22 regions between $\mathrm{B} 73$ and A188, Mo17 and A188, and W22 and A188, respectively 
1 (Table 3). Moreover, 17,168, 17,634 and 17,382 A188 genes showed no variations in

2 CDS and intron regions as compared with B73, Mo17 and W22, respectively (Table

3 3). In particular, as compared with B73, Mo17 and W22, 8,647, 9,054 and 8,854

4 genes were highly conserved without any genetic variation (including $2 \mathrm{~kb}$ upstream

5 and downstream), respectively (Table 3). Moreover, we found 23,989, 24,424 and

6 20,860 A188 genes showed synonymous variations in CDS compared to B73, Mo17,

7 and W22, respectively (Table 3). Compared with B73, 22,958, 21,975 and 7,210

8 genes in A188 resulted in amino acid changes, missense mutation and non-frameshift

9 InDels, respectively (Table 3). Mapped to Mo17, 23,313, 21,601 and 7,257 genes in

10 A188 were identified to contain amino acid changes, missense mutation in CDS and

11 non-frameshift InDels, respectively (Table 3). Aligned to W22, 23,070, 21,869 and

127,295 A188 genes showed amino acid changes, missense mutation in CDS and

13 non-frameshift InDels, respectively (Table 3). All of these genes were classified as

14 structurally conserved genes between A188 and the other lines, which accounted for $\geq$

$1568.61 \%$ of the annotated A188 genes and may function in basic physiological effects.

16 By comparing B73 and A188 genomes, we identified 737, 506, 841, 1,671,

1710,834 and 2,362 genes in A188 that generated start codon mutations, stop codon

18 mutations, splice donor mutations, splice acceptor mutations, frameshift InDel in CDS

19 and premature termination codon mutations, respectively (Table 3). Similarly, 747,

$20504,801,1,559,10,982$ and 2,355 genes in A188 led to start codon mutations, stop

21 codon mutations, splice donor mutations, splice acceptor mutations, frameshift InDels

22 in CDS and premature termination codon mutations, respectively, as compared with 
1 Mo17 (Table 3). As well, 742, 506, 811, 1,486, 10,889 and 2,389 genes in A188

2 showed start codon mutations, stop codon mutations, splice donor mutations, splice

3 acceptor mutations, frameshift InDels in CDS and premature termination codon

4 mutations, as compared with W22, respectively (Table 3). In addition, 204, 262 and

5228 PAV genes were identified between A188 and the lines B73, Mo17 and W22

6 genomes, respectively (Table 3). Finally, a total of 13,224 (29.62\%), 13,306

$7 \quad(29.80 \%)$ and 13,167 (29.49\%) A188 genes had large structural variations (start- or

8 stop-codon mutations, splice-donor or splice-acceptor mutations, frameshift mutations,

9 premature termination codon mutations or PAV variations) as compared with B73,

10 Mo17 and W22 genomes, respectively.

\section{A188 genome-based genetic dissection of tissue culture response}

12 Recently, by using the $\mathrm{F}_{3: 4}$ population derived from B73 and WCIC2 (a near isogenic

13 line of B73 containing several A188 segments), a locus associated with embryogenic

14 and regenerative capabilities of immature embryo was fine-mapped within a $3.06 \mathrm{M}$

15 region (chr3:178772856-181826658) based on the B73 reference genome, suggesting

16 that the genes harbored by the A188 segment caused the high callus formation ratio

17 (Salvo et al., 2018).

18 To explore the candidate genes of embryonic callus induction, we aligned the

19 3.06 M B73 segment to the A188 genome and revealed a 3.89 M syntenic segment

20 (Fig. 3) in A188. Within the $3.89 \mathrm{M}$ segment, 51, 57, and 57 A188 genes were

21 identified syntenic to B73, Mo17 and W22 syntenic segment, respectively (Table

22 S10). Among them, 6, 14, and 6 genes showed large structural variation (LSV: 
1 premature termination codon, stop codon loss, frameshift deletion, or frameshift

2 insertion) relative to B73, Mo17 and W22, respectively (Table S11), and 4 LSV genes

3 in A188 were simultaneously identified in comparison with the other 3 lines (Table

$44)$.

5 We also focused on the nonsyntenic genes of A188 in the QTL. Finally, we

6 identified 48, 42, and 42 A188 genes in the QTL interval that were identified

7 nonsyntenic, as compared with B73, Mo17 and W22 genomes, respectively (Table

8 S10). To further identify whether the nonsyntenic genes have homologues in other

9 sites of the 3 inbred lines, we mapped these nonsyntenic genes to the B73, MO17 and

10 W22 whole genomes. Finally, 28, 11, and 24 A188 nonsyntenic genes showed LSV to

11 their corresponding homologues in B73, Mo17 and W22, respectively (Table S11),

12 and 4 LSV genes in A188 were simultaneously identified in comparison with the

13 other 3 inbred lines (Table 4).

14 Moreover, previous studies have demonstrated that changes in gene expression

15 can be induced during somatic embryogenesis to respond to tissue culture process (Ge

16 et al., 2017; Salvo et al., 2018; Shen et al., 2012; Zhang et al., 2014). Based on the

17 reported transcriptome data of A188 (Zhang et al., 2014), four of the 99 A188 genes

18 within the mapped QTL were regulated by more than 8 folds in different stages of

19 immature embryo culture, relative to control. Collectively, the 4 syntenic genes with

20 LSV, the 4 nonsyntenic genes with LSV as well as the 4 differentially expressed

21 genes were designated as the candidate genes responsible for tissue culture capability

22 of A188 immature embryo in this study (Table 4). 


\section{Discussion}

2 Although A188 is limited from the application in breeding programs, due to its poor

3 agronomic traits, A188 shows significant phenotypic variations from B73, Mo17 and

4 W22, including plant height, tassel branch number, ear number, protein concentration,

5 days to tassel, days to silk etc., especially EC induction ratio (Table 1). The

6 phenotypic performance is determined by the combination of genotype and

7 environment. To better understand the mechanisms underlying the phenotypic

8 difference between A188, B73, Mo17 and W22, we sequenced and de novo

9 assembled the A188 genome. Finally, we assembled the A188 genome into 2,207.74

$10 \mathrm{Mb}$ with a scaffold $\mathrm{N} 50$ size of $11.61 \mathrm{Mb}$ (Table 2, Table S2). As expected, A188

11 showed large genomic variations as compared with B73, Mo17 and W22 (Fig. 2,

12 Table 3, Tables S7, S8 and S9). Our new A188 genome provides a good resource to

13 map causal genes controlling these various traits. We also identified a number of

14 A188 genes presenting structure variations relative to other 3 inbred lines, such as

15 genes with start/stop codon mutations, splice donor/acceptor mutations and frameshift

16 InDels, which provides a novel view to study gene function and evolutionary analysis.

17 EC induction from maize immature embryo is highly dependent on genotype,

18 which resulted in only a few functional genes identified. Accordingly, the molecular

19 mechanism underlying EC induction still remains unclear in maize. Combined our

20 new A188 genome, previously reported QTLs, and RNA sequencing data, we

21 successfully identified 12 candidate genes responsible for maize tissue culture

22 response (Table 4). These candidate genes provide new insight into understanding the 
1 molecular mechanisms of maize tissue culture response, and provide new gene

2 resources for improving maize embryonic callus induction and maize genetic

3 transformation, which will further contribute to gene function revelation and

4 transgenic breeding in maize. Especially, ZmY09GFa037173 showed a premature

5 termination mutation in A188, which was annotated as an Ankyrin repeat-containing

6 protein and involved in signal transduction. In addition, the Arabidopsis homologue,

7 Itn1, was previously reported to regulate ROS accumulation under salt-stress through

8 regulating ABA signaling pathways (Sakamoto et al., 2008), which suggest that

9 ZmY09GFa037173 have a potential to induce maize callus formation by mediating

10 ROS levels.

11 Owing to the vast genetic diversity among maize germplasms, the currently

12 identified genetic variants by comparison of nine public maize genomes are still

13 unsaturated (Yang et al., 2019). The previously study suggest that more than 20

14 reference genomes of maize and teosinte were required for performing pan-genome

15 construction, which will provide better coverage for genetic variations of the Zea

16 genus (Yang et al., 2019). Our new sequenced and assembled A188 genome thereby

17 provides a new reference genome and structure variation resource.

\section{Supplementary data}

19 Fig. S1. Code gene function annotation result using the public databases of NR,

20 Swiss-Prot, eggNOG, GO and KEGG.

21 Fig. S2. Whole-genome comparison of A188 versus B73 and Mo17. Grey lines

22 represent the one-to-one aligned genes between each pair of pseudomolecules. 
1 Fig. S3. Histogram of InDel number comparisons of A188 versus B73 and Mo17 of

2 the whole genome and coding regions.

3 Fig. S4. Length distribution of PAV sequences between A188 and B73 genomes (a),

$4 \quad$ A188 and Mo17 genomes (b), A188 and W22 genomes (c).

5 Table S1. Summary of sequencing data of A188 genome.

6 Table S2. Details of the A188 genome assembly.

7 Table S3. Details of the 10 A188 pseudo-chromosomes.

8 Table S4. BUSCO analysis.

9 Table S5. Comparisons of repetitive elements between A188, B73 and Mo17.

10 Table S6. Statistics of A188, B73 and Mo17 gene models.

11 Table S7. Summary of aligned sequences, SNPs and InDels in A188, B73, Mo17 and

12 W22 genomes.

13 Table S8. Genome distribution of SNPs and InDels between A188, B73, Mo17 and

14 W22 genomes.

15 Table S9. Summary of PAV genes in A188, B73, Mo17 and W22 genomes.

16 Table S10. Syntenic analysis of the 99 genes on the A188 syntenic segment

17 compared with B73, Mo17 and W22 syntenic segments.

18 Table S11. Mutation type of the 99 genes within the QTL on the A188 syntenic

19 segment compared with B73, Mo17 and W22 homologous genes.

\section{Acknowledgements}

21 This study was supported by the National Natural Science Foundation of China 22 (31871637 and 32072073), and the Project of Transgenic New Variety Cultivation 
1 (2016ZX08003003).

\section{Author contributions}

3 Y.S., F.G. and L.M. designed the research. J.Q., P.L. and J.H. performed genome

4 assembly, genome annotation and genome comparison. F.G. L.P. and J.H. prepared

5 DNA/RNA samples and constructed the next-generation-sequencing library. P.L.

6 performed phenotype investigation. Y.S., F.G., L.M., J.Q., P.L., L.P., J.H., C.Z., G.Y.,

7 C.Y., S.G., and G.P. participated in the analysis. F.G. J.Q., P.L., L.P., L.M. and Y.S.

8 wrote and revised the manuscript.

\section{Data Availability}

10 All datasets reported in this study have been deposited in GenBank (NCBI) with the

11 following accession IDs: Genome assembly, JADZIA000000000; Raw data for

12 genome assembly and gene annotation, PRJNA678284.

\section{Conflict of Interest Statement}

14 The authors declare no conflict of interests.

\section{Reference}

Armstrong C, Romero-Severson J, Hodges T. 1992. Improved tissue culture response of an elite maize inbred through backcross breeding, and identification of chromosomal regions important for regeneration by RFLP analysis. Theoretical and applied genetics 84, 755-762.

Bronsema F, Van Oostveen W, Van Lammeren A. 1997. Comparative analysis of callus formation and regeneration on cultured immature maize embryos of the inbred lines A188 and A632. Plant cell, tissue and organ culture 50, 57-65.

Brown PJ, Upadyayula N, Mahone GS, Tian F, Bradbury PJ, Myles S, Holland JB, Flint-Garcia S, McMullen MD, Buckler ES. 2011. Distinct genetic architectures for male and female inflorescence traits of maize. PLoS genetics 7, e1002383. 
Buckler ES, Gaut BS, McMullen MD. 2006. Molecular and functional diversity of maize. Current opinion in plant biology 9, 172-176.

Cantarel BL, Korf I, Robb SM, Parra G, Ross E, Moore B, Holt C, Alvarado AS, Yandell M. 2008. MAKER: an easy-to-use annotation pipeline designed for emerging model organism genomes. Genome research 18, 188-196.

Chaisson MJ, Tesler G. 2012. Mapping single molecule sequencing reads using basic local alignment with successive refinement (BLASR): application and theory. BMC Bioinformatics 13, 238.

Cook JP, McMullen MD, Holland JB, Tian F, Bradbury P, Ross-Ibarra J, Buckler ES, Flint-Garcia SA. 2011. Genetic architecture of maize kernel composition in the nested association mapping and inbred association panels. Plant physiology, pp. 111.185033.

Frame BR, McMurray JM, Fonger TM, Main ML, Taylor KW, Torney FJ, Paz MM, Wang K. 2006. Improved Agrobacterium-mediated transformation of three maize inbred lines using MS salts. Plant cell reports 25, 1024-1034.

Frame BR, Shou H, Chikwamba RK, Zhang Z, Xiang C, Fonger TM, Pegg SEK, Li B, Nettleton DS, Pei D, Wang K. 2002. Agrobacterium tumefaciens-Mediated Transformation of Maize Embryos Using a Standard Binary Vector System. Plant physiology 129, 13-22.

Gacheri P, Machuka J, Ombori O, Bukachi B. 2015. Agrobacterium mediated transformation of selected maize inbred lines with pPZP200 towards enhancment of lysine and methionine content. Journal of Biology, Agriculture and Healthcare 5.

Ge F, Hu H, Huang X, Zhang Y, Wang Y, Li Z, Zou C, Peng H, Li L, Gao S. 2017. Metabolomic and Proteomic Analysis of Maize Embryonic Callus induced from immature embryo. Scientific Reports 7, 1004.

Green C, Phillips R. 1975. Plant Regeneration from Tissue Cultures of Maize 1. Crop Science 15, 417-421.

Hodges T, Kamo K, Imbrie C, Becwar M. 1986. Genotype specificity of somatic embryogenesis and regeneration in maize. Bio/technology 4, 219-223.

Ishida Y, Saito H, Ohta S, Hiei Y, Komari T, Kumashiro T. 1996. High efficiency transformation of maize (Zea mays L.) mediated by Agrobacterium tumefaciens. Nature Biotechnology 14, 745-750.

Jiao Y, Peluso P, Shi J, Liang T, Stitzer MC, Wang B, Campbell MS, Stein JC, 
Wei X, Chin C-S, Guill K, Regulski M, Kumari S, Olson A, Gent J, Schneider KL, Wolfgruber TK, May MR, Springer NM, Antoniou E, McCombie WR, Presting GG, McMullen M, Ross-Ibarra J, Dawe RK, Hastie A, Rank DR, Ware D. 2017. Improved maize reference genome with single-molecule technologies. Nature 546, 524.

Keller O, Kollmar M, Stanke M, Waack S. 2011. A novel hybrid gene prediction method employing protein multiple sequence alignments. Bioinformatics 27, 757-763.

Krakowsky M, Lee M, Garay L, Woodman-Clikeman W, Long M, Sharopova N, Frame B, Wang K. 2006. Quantitative trait loci for callus initiation and totipotency in maize (Zea mays L.). Theoretical and applied genetics 113, 821-830.

Kurtz S, Phillippy A, Delcher AL, Smoot M, Shumway M, Antonescu C, Salzberg SL. 2004. Versatile and open software for comparing large genomes. Genome biology 5, R12.

Lai J, Li R, Xu X, Jin W, Xu M, Zhao H, Xiang Z, Song W, Ying K, Zhang M. 2010. Genome-wide patterns of genetic variation among elite maize inbred lines. Nature genetics 42, 1027.

Li C, Xiang X, Huang Y, Zhou Y, An D, Dong J, Zhao C, Liu H, Li Y, Wang Q, Du C, Messing J, Larkins BA, Wu Y, Wang W. 2020. Long-read sequencing reveals genomic structural variations that underlie creation of quality protein maize. Nature communications 11, 17.

Li H. 2013. Aligning sequence reads, clone sequences and assembly contigs with BWA-MEM. arXiv preprint arXiv:1303.3997.

Lowe BA, Way MM, Kumpf JM, Rout J, Warner D, Johnson R, Armstrong CL, Spencer MT, Chomet PS. 2006. Marker assisted breeding for transformability in maize. Molecular Breeding 18, 229-239.

Lowe K, Wu E, Wang N, Hoerster G, Hastings C, Cho MJ, Scelonge C, Lenderts B, Chamberlin M, Cushatt J, Wang L, Ryan L, Khan T, Chow-Yiu J, Hua W, Yu M, Banh J, Bao Z, Brink K, Igo E, Rudrappa B, Shamseer PM, Bruce W, Newman L, Shen B, Zheng P, Bidney D, Falco C, Register J, Zhao ZY, Xu D, Jones T, Gordon-Kamm W. 2016. Morphogenic Regulators Baby boom and Wuschel Improve Monocot Transformation. Plant Cell 28, 1998-2015.

Pan G, Zhang Z, Wei X, Song Y, Zhao M, Xia Y, Rong T. 2006. QTL analysis of maize (Zea mays L.) embryo culturing capacity. Zuo wu xue bao 32, 7-13. 
Peiffer JA, Romay MC, Gore MA, Flint-Garcia SA, Zhang Z, Millard MJ, Gardner CA, McMullen MD, Holland JB, Bradbury PJ. 2014. The genetic architecture of maize height. Genetics 196, 1337-1356.

Pendleton M, Sebra R, Pang AWC, Ummat A, Franzen O, Rausch T, Stütz AM, Stedman W, Anantharaman T, Hastie A, Dai H, Fritz MH-Y, Cao H, Cohain A, Deikus G, Durrett RE, Blanchard SC, Altman R, Chin C-S, Guo Y, Paxinos EE, Korbel JO, Darnell RB, McCombie WR, Kwok P-Y, Mason CE, Schadt EE, Bashir A. 2015. Assembly and diploid architecture of an individual human genome via single-molecule technologies. Nature Methods 12, 780.

Sakamoto H, Matsuda O, Iba K. 2008. ITN1, a novel gene encoding an ankyrin-repeat protein that affects the ABA-mediated production of reactive oxygen species and is involved in salt-stress tolerance in Arabidopsis thaliana. Plant J 56, 411-422.

Salvo S, Cook J, Carlson AR, Hirsch CN, Kaeppler SM, Kaeppler HF. 2018. Genetic Fine-Mapping of a Quantitative Trait Locus (QTL) Associated with Embryogenic Tissue Culture Response and Plant Regeneration Ability in Maize (Zea mays L.). The Plant Genome.

Schnable PS, Ware D, Fulton RS, Stein JC, Wei F, Pasternak S, Liang C, Zhang J, Fulton L, Graves TA. 2009. The B73 maize genome: complexity, diversity, and dynamics. science 326, 1112-1115.

Shen Y, Jiang Z, Yao X, Zhang Z, Lin H, Zhao M, Liu H, Peng H, Li S, Pan G. 2012. Genome expression profile analysis of the immature maize embryo during dedifferentiation. PLoS ONE 7.

Simão FA, Waterhouse RM, Ioannidis P, Kriventseva EV, Zdobnov EM. 2015. BUSCO: assessing genome assembly and annotation completeness with single-copy orthologs. Bioinformatics 31, 3210-3212.

Springer NM, Anderson SN, Andorf CM, Ahern KR, Bai F, Barad O, Barbazuk WB, Bass HW, Baruch K, Ben-Zvi G, Buckler ES, Bukowski R, Campbell MS, Cannon EKS, Chomet P, Dawe RK, Davenport R, Dooner HK, Du LH, Du C, Easterling KA, Gault C, Guan J-C, Hunter CT, Jander G, Jiao Y, Koch KE, Kol G, Köllner TG, Kudo T, Li Q, Lu F, Mayfield-Jones D, Mei W, McCarty DR, Noshay JM, Portwood JL, Ronen G, Settles AM, Shem-Tov D, Shi J, Soifer I, Stein JC, Stitzer MC, Suzuki M, Vera DL, Vollbrecht E, Vrebalov JT, Ware D, 
Wei S, Wimalanathan K, Woodhouse MR, Xiong W, Brutnell TP. 2018. The maize W22 genome provides a foundation for functional genomics and transposon biology. Nature genetics 50, 1282-1288.

Springer NM, Ying K, Fu Y, Ji T, Yeh C-T, Jia Y, Wu W, Richmond T, Kitzman J, Rosenbaum H. 2009. Maize inbreds exhibit high levels of copy number variation $(\mathrm{CNV})$ and presence/absence variation (PAV) in genome content. PLoS genetics 5, e1000734.

Sun S, Zhou Y, Chen J, Shi J, Zhao H, Zhao H, Song W, Zhang M, Cui Y, Dong X, Liu H, Ma X, Jiao Y, Wang B, Wei X, Stein JC, Glaubitz JC, Lu F, Yu G, Liang C, Fengler K, Li B, Rafalski A, Schnable PS, Ware DH, Buckler ES, Lai J. 2018. Extensive intraspecific gene order and gene structural variations between Mo17 and other maize genomes. Nat Genet 50, 1289-1295.

Tello-Ruiz MK, Stein J, Wei S, Preece J, Olson A, Naithani S, Amarasinghe V, Dharmawardhana P, Jiao Y, Mulvaney J. 2015. Gramene 2016: comparative plant genomics and pathway resources. Nucleic acids research 44, D1133-D1140.

Wang B, Tseng E, Regulski M, Clark TA, Hon T, Jiao Y, Lu Z, Olson A, Stein JC, Ware D. 2016. Unveiling the complexity of the maize transcriptome by single-molecule long-read sequencing. Nature communications 7, 11708.

Xu L, Zhang Y, Su Y, Liu L, Yang J, Zhu Y, Li C. 2010. Structure and evolution of full-length LTR retrotransposons in rice genome. Plant systematics and evolution 287, 19-28.

Yang N, Liu J, Gao Q, Gui S, Chen L, Yang L, Huang J, Deng T, Luo J, He L, Wang Y, Xu P, Peng Y, Shi Z, Lan L, Ma Z, Yang X, Zhang Q, Bai M, Li S, Li W, Liu L, Jackson D, Yan J. 2019. Genome assembly of a tropical maize inbred line provides insights into structural variation and crop improvement. Nature genetics $\mathbf{5 1}$, 1052-1059.

Zhang X, Salvo SAGD, Hirsch CN, Buell CR, Kaeppler SM, Kaeppler HF. 2014. Whole Transcriptome Profiling of Maize during Early Somatic Embryogenesis Reveals Altered Expression of Stress Factors and Embryogenesis-Related Genes. PLOS ONE 9. 


\section{Tables}

Table 1 The phenotypic performances of agronomic traits among different inbred lines

\begin{tabular}{cccccccccccccc}
\hline Line & ECIR $^{\#}$ & Sig. & Plant height* & Sig. & TBN* $^{*}$ & Sig. & Ear No.* & Sig. & Pro con. & Sig. & DtT & DtP & DtS \\
\hline A188 & $91.53 \% \pm 5.55 \%$ & a & $126.00 \pm 16.83$ & d & $12.36 \pm 2.71$ & a & $1.38 \pm 0.62$ & a & $11.98 \pm 0.04$ & b & 51 & 59 & 61 \\
W22 & $1.67 \% \pm 1.85$ & c & $159.50 \pm 17.03$ & c & $11.48 \pm 2.48$ & a & $1.29 \pm 0.60$ & ab & $10.48 \pm 0.07$ & c & 67 & 70 & 70 \\
Mo17 & $6.94 \% \pm 1.39$ & b & $184.29 \pm 17.99$ & b & $6.21 \pm 1.05$ & b & $1.07 \pm 0.68$ & bc & $12.59 \pm 0.11$ & a & 70 & 71 & 72 \\
B73 & $0 \pm 0$ & c & $203.36 \pm 13.92$ & a & $6.64 \pm 1.23$ & b & $0.92 \pm 0.51$ & c & $9.34 \pm 0.05$ & d & 71 & 72 & 73 \\
\hline
\end{tabular}

The results are shown as the means $\pm \mathrm{SD}$ (“*” $\mathrm{n}=42$, “\#” $\mathrm{n}=3$ ); “ $\mathrm{a}, \mathrm{b}, \mathrm{c}, \mathrm{d}$ ” represents the significant differences of trait with $P<0.05$. ECIR: embryonic callus induction ratio; Sig.: significance; TBN:

Tassel branch numbers; Pro con.: protein concentration; DtT: Days to Tassel; DtP: Days to Pollination;

DtS: Days to Silk.

Table 2 Global statistics for the A188 genome assembly

\begin{tabular}{cccc}
\hline & PacBio assembly & Pacbio+Bionano hybrid & Pseudomolecule \\
& & assembly & $2,084.35$ \\
Total length of assembly (Mb) & $2,127.72$ & $2,210.33$ & - \\
Nong size (Mb) & 1.06 & 11.61 & - \\
Number of sequences & 4.97 & 47.84 & 10 \\
\hline
\end{tabular}


Table 3 Variations within genes between A188, B73, Mo17 and W22 genomes

\begin{tabular}{|c|c|c|c|}
\hline Variation type & A188 to B73 & A188 to Mo17 & A188 to W22 \\
\hline 1. Structurally conserved genes & 30635 & 31095 & 30764 \\
\hline No DNA variation in CDS & 20557 & 21007 & 20713 \\
\hline No DNA variation in CDS and intron & 17168 & 17634 & 17382 \\
\hline No DNA variation in genic region* & 8647 & 9054 & 8854 \\
\hline Without amino acid substitutions & 23989 & 24424 & 20860 \\
\hline With amino acid changes & 22958 & 23313 & 23070 \\
\hline With missense mutation in CDS & 21975 & 21601 & 21869 \\
\hline With 3N InDel in CDS & 7210 & 7257 & 7295 \\
\hline 2. Genes with large structural mutations & 13020 & 13044 & 12939 \\
\hline Start codon mutation & 737 & 747 & 742 \\
\hline Stop codon mutation & 506 & 504 & 506 \\
\hline Splice donor mutation & 841 & 801 & 811 \\
\hline Splice acceptor mutation & 1671 & 1559 & 1486 \\
\hline With $3 \mathrm{~N} \pm 1 \mathrm{InDel}$ in CDS & 10834 & 10982 & 10889 \\
\hline Premature termination codon & 2362 & 2355 & 2389 \\
\hline 3. PAV genes & 204 & 262 & 228 \\
\hline A188 present PAV genes & 100 & 116 & 112 \\
\hline A188 absent PAV genes & 104 & 146 & 116 \\
\hline Total of genes with large structural variations & 13224 & 13306 & 13167 \\
\hline
\end{tabular}

* Genic regions include the $2 \mathrm{~kb}$ upstream and downstream of the gene body. 
Table 4 Tissue culture response candidate genes

\begin{tabular}{|c|c|c|c|c|c|c|c|c|}
\hline $\begin{array}{c}\text { A188 Gene } \\
\text { ID }\end{array}$ & $\begin{array}{c}\text { B73 } \\
\text { homologous }\end{array}$ & $\begin{array}{l}\text { Mutation } \\
\text { type to B73 }\end{array}$ & $\begin{array}{c}\text { Mo17 } \\
\text { homologous }\end{array}$ & $\begin{array}{l}\text { mutation } \\
\text { type to } \\
\text { Mo17 }\end{array}$ & $\begin{array}{l}\text { W22 } \\
\text { homologous }\end{array}$ & $\begin{array}{l}\text { mutation } \\
\text { type to } \\
\text { W22 }\end{array}$ & $\begin{array}{l}\text { Homologous } \\
\text { type }\end{array}$ & Annotation \\
\hline $\begin{array}{l}\text { ZmY09GF } \\
\text { a037173 }\end{array}$ & $\begin{array}{c}\text { GRMZM2G } \\
123977\end{array}$ & stop gain & $\begin{array}{c}\mathrm{Zm} 00014 \mathrm{a} 0 \\
19537\end{array}$ & stop gain & $\begin{array}{c}\mathrm{Zm00004b0} \\
18533\end{array}$ & stop gain & $\begin{array}{l}\text { syntenic } \\
\text { gene }\end{array}$ & $\begin{array}{l}\text { ankyrin } \\
\text { repeat-containing } \\
\text { protein;signal } \\
\text { transduction }\end{array}$ \\
\hline $\begin{array}{l}\text { ZmY09GF } \\
\text { a037487 }\end{array}$ & $\begin{array}{c}\text { GRMZM2G } \\
359234\end{array}$ & $\begin{array}{l}\text { frameshift } \\
\text { deletion }\end{array}$ & $\begin{array}{c}\mathrm{Zm} 00014 \mathrm{a} 0 \\
19543\end{array}$ & $\begin{array}{l}\text { stoploss } \\
\text { and } \\
\text { frameshift } \\
\text { deletion }\end{array}$ & $\begin{array}{c}\mathrm{Zm00004b0} \\
18529\end{array}$ & $\begin{array}{l}\text { frameshift } \\
\text { deletion }\end{array}$ & $\begin{array}{l}\text { syntenic } \\
\text { gene }\end{array}$ & $\begin{array}{l}\text { UDP-glucuronic } \\
\text { acid decarboxylase }\end{array}$ \\
\hline $\begin{array}{l}\text { ZmY09GF } \\
\text { a038636 }\end{array}$ & $\begin{array}{c}\text { GRMZM2G } \\
337905\end{array}$ & stop gain & $\begin{array}{c}\mathrm{Zm} 00014 \mathrm{a} 0 \\
19529\end{array}$ & stop gain & $\begin{array}{c}\mathrm{Zm00004b0} \\
18516\end{array}$ & stop gain & $\begin{array}{l}\text { syntenic } \\
\text { gene }\end{array}$ & $\begin{array}{l}\text { helicase-like } \\
\text { protein;DNA repair }\end{array}$ \\
\hline $\begin{array}{l}\text { ZmY09GF } \\
\text { a039738 }\end{array}$ & $\begin{array}{c}\text { GRMZM5G } \\
856598\end{array}$ & stop gain & $\begin{array}{c}\mathrm{Zm} 00014 \mathrm{a} 0 \\
39033\end{array}$ & stop gain & $\begin{array}{c}\mathrm{Zm00004b0} \\
18443\end{array}$ & stop gain & $\begin{array}{l}\text { syntenic } \\
\text { gene }\end{array}$ & $\begin{array}{l}\text { Probable anion } \\
\text { transporter }\end{array}$ \\
\hline $\begin{array}{l}\text { ZmY09GF } \\
\text { a035987 }\end{array}$ & $\begin{array}{c}\text { GRMZM2G } \\
341918\end{array}$ & $\begin{array}{l}\text { frameshift } \\
\text { insertion and } \\
\text { stop gain }\end{array}$ & $\begin{array}{c}\mathrm{Zm} 00014 \mathrm{a} 0 \\
13928\end{array}$ & stop gain & $\begin{array}{c}\mathrm{Zm00004b0} \\
00208\end{array}$ & $\begin{array}{l}\text { frameshift } \\
\text { insertion } \\
\text { and stop } \\
\text { gain }\end{array}$ & $\begin{array}{l}\text { nonsyntenic } \\
\text { homologous }\end{array}$ & $\begin{array}{c}\text { zinc finger } \\
\text { MYM-type protein } \\
\text { 1-like }\end{array}$ \\
\hline $\begin{array}{l}\text { ZmY09GF } \\
\text { a037580 }\end{array}$ & $\begin{array}{c}\text { GRMZM2G } \\
156296\end{array}$ & $\begin{array}{l}\text { frameshift } \\
\text { insertion and } \\
\text { stop gain }\end{array}$ & $\begin{array}{c}\mathrm{Zm} 00014 \mathrm{a} 0 \\
10023\end{array}$ & $\begin{array}{l}\text { frameshift } \\
\text { insertion }\end{array}$ & $\begin{array}{c}\mathrm{Zm} 00004 \mathrm{~b} 0 \\
30624\end{array}$ & $\begin{array}{l}\text { frameshift } \\
\text { insertion } \\
\text { and stop } \\
\text { gain }\end{array}$ & $\begin{array}{l}\text { nonsyntenic } \\
\text { homologous }\end{array}$ & $\begin{array}{l}\text { uncharacterized } \\
\text { protein } \\
\text { loc } 103635851\end{array}$ \\
\hline $\begin{array}{l}\text { ZmY09GF } \\
\text { a038110 }\end{array}$ & $\begin{array}{c}\text { GRMZM2G } \\
084717\end{array}$ & $\begin{array}{l}\text { frameshift } \\
\text { deletion }\end{array}$ & $\begin{array}{c}\mathrm{Zm} 00014 \mathrm{a} 0 \\
20349\end{array}$ & $\begin{array}{l}\text { frameshift } \\
\text { deletion }\end{array}$ & $\begin{array}{c}\mathrm{Zm00004b0} \\
17917\end{array}$ & $\begin{array}{l}\text { frameshift } \\
\text { deletion }\end{array}$ & $\begin{array}{l}\text { nonsyntenic } \\
\text { homologous }\end{array}$ & hypothetical protein \\
\hline $\begin{array}{c}\text { ZmY09GF } \\
\text { a038645 }\end{array}$ & $\begin{array}{c}\text { GRMZM2G } \\
078468\end{array}$ & stop gain & $\begin{array}{c}\mathrm{Zm} 00014 \mathrm{a} 0 \\
04443\end{array}$ & stop gain & $\begin{array}{c}\mathrm{Zm00004b0} \\
21555\end{array}$ & $\begin{array}{l}\text { frameshift } \\
\text { deletion } \\
\text { and stop }\end{array}$ & $\begin{array}{l}\text { nonsyntenic } \\
\text { homologous }\end{array}$ & hypothetical protein \\
\hline
\end{tabular}




\begin{tabular}{|c|c|c|c|c|c|c|c|c|}
\hline $\begin{array}{l}\text { ZmY09GF } \\
\text { a036216 }\end{array}$ & $\begin{array}{l}\text { GRMZM2G } \\
091445\end{array}$ & - & $\begin{array}{c}\mathrm{Zm} 00014 \mathrm{a} 0 \\
20366\end{array}$ & $\begin{array}{l}\text { synonymo } \\
\text { us SNV }\end{array}$ & $\begin{array}{c}\text { Zm00004b0 } \\
17905\end{array}$ & - & $\begin{array}{c}\text { DE gene, } \\
\text { nonsyntenic } \\
\text { homologous }\end{array}$ & $\begin{array}{l}\text { Defensin-like } \\
\text { protein }\end{array}$ \\
\hline $\begin{array}{l}\text { ZmY09GF } \\
\text { a038775 }\end{array}$ & $\begin{array}{l}\text { GRMZM2G } \\
084779\end{array}$ & $\begin{array}{l}\text { synonymous } \\
\text { SNV }\end{array}$ & $\begin{array}{c}\mathrm{Zm} 00014 \mathrm{a} 0 \\
20354\end{array}$ & $\begin{array}{l}\text { synonymo } \\
\text { us SNV }\end{array}$ & $\begin{array}{c}\text { Zm00004b0 } \\
17916\end{array}$ & - & $\begin{array}{c}\text { DE gene, } \\
\text { nonsyntenic } \\
\text { homologous }\end{array}$ & $\begin{array}{c}\text { potasium ion uptake } \\
\text { permease }\end{array}$ \\
\hline $\begin{array}{l}\text { ZmY09GF } \\
\text { a036902 }\end{array}$ & $\begin{array}{c}\text { AC209784.3 } \\
\text { _FG007 }\end{array}$ & $\begin{array}{l}\text { synonymous } \\
\text { SNV }\end{array}$ & $\begin{array}{c}\mathrm{Zm} 00014 \mathrm{a} 0 \\
19526\end{array}$ & $\begin{array}{l}\text { synonymo } \\
\text { us SNV }\end{array}$ & $\begin{array}{c}\mathrm{Zm} 00004 \mathrm{~b} 0 \\
18512\end{array}$ & $\begin{array}{l}\text { synonymo } \\
\text { us SNV }\end{array}$ & $\begin{array}{l}\text { DE gene, } \\
\text { syntenic } \\
\text { gene }\end{array}$ & $\begin{array}{c}\text { Probable mediator } \\
\text { of RNA polymerase } \\
\text { II transcription } \\
\text { subunit 37c; MAPK } \\
\text { signaling pathway; } \\
\text { Estrogen signaling } \\
\text { pathway }\end{array}$ \\
\hline $\begin{array}{l}\text { ZmY09GF } \\
\text { a039032 }\end{array}$ & $\begin{array}{c}\text { GRMZM2G } \\
065557\end{array}$ & - & $\begin{array}{c}\mathrm{Zm} 00014 \mathrm{a} 0 \\
36794\end{array}$ & - & $\begin{array}{c}\text { Zm00004b0 } \\
18459\end{array}$ & - & $\begin{array}{c}\text { DE gene, } \\
\text { syntenic } \\
\text { gene }\end{array}$ & hypothetical protein \\
\hline
\end{tabular}

DE gene: differentially expressed genes during tissue culture response.

\section{Figure Legends}

Fig. 1 Overview of the trait difference between A188, B73, Mo17 and W22 inbred

lines, including plant height $(\mathbf{A})$, ear trait $(\mathbf{B})$, and kernel size $(\mathbf{C})$.

Fig. 2 Features of the A188 genome. a, Transposable-element density; b, gene density;

c, $\mathbf{d}$ and e, numbers of PAVs (c), SNPs (d) and InDels (e) compared with B73 genome.

The sliding window is $1 \mathrm{Mb}$ for all tracks.

Fig. 3 Tissue culture response candidate loci. The 3.89 M A188 segment (QTL for maize tissue culture response) aligned to the syntenic segment of B73, Mo17 and W22 genomes. The red, green and blue lines indicate aligned A188 genes in the 3.89 M segment to the B73, Mo17 and W22, respectively. 


\section{Figures}
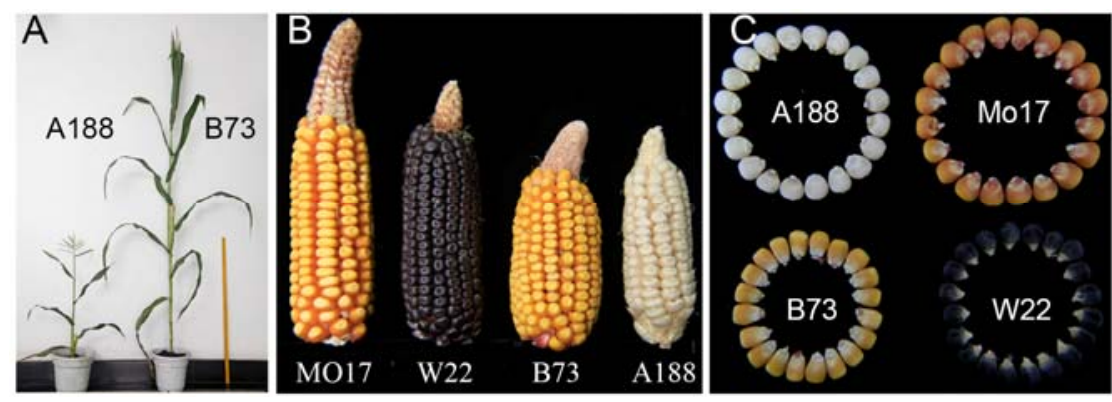

Fig. 1 Overview of the trait difference between A188, B73, Mo17 and W22 inbred

lines, including plant height (A), ear trait $(\mathbf{B})$, and kernel size $(\mathbf{C})$.

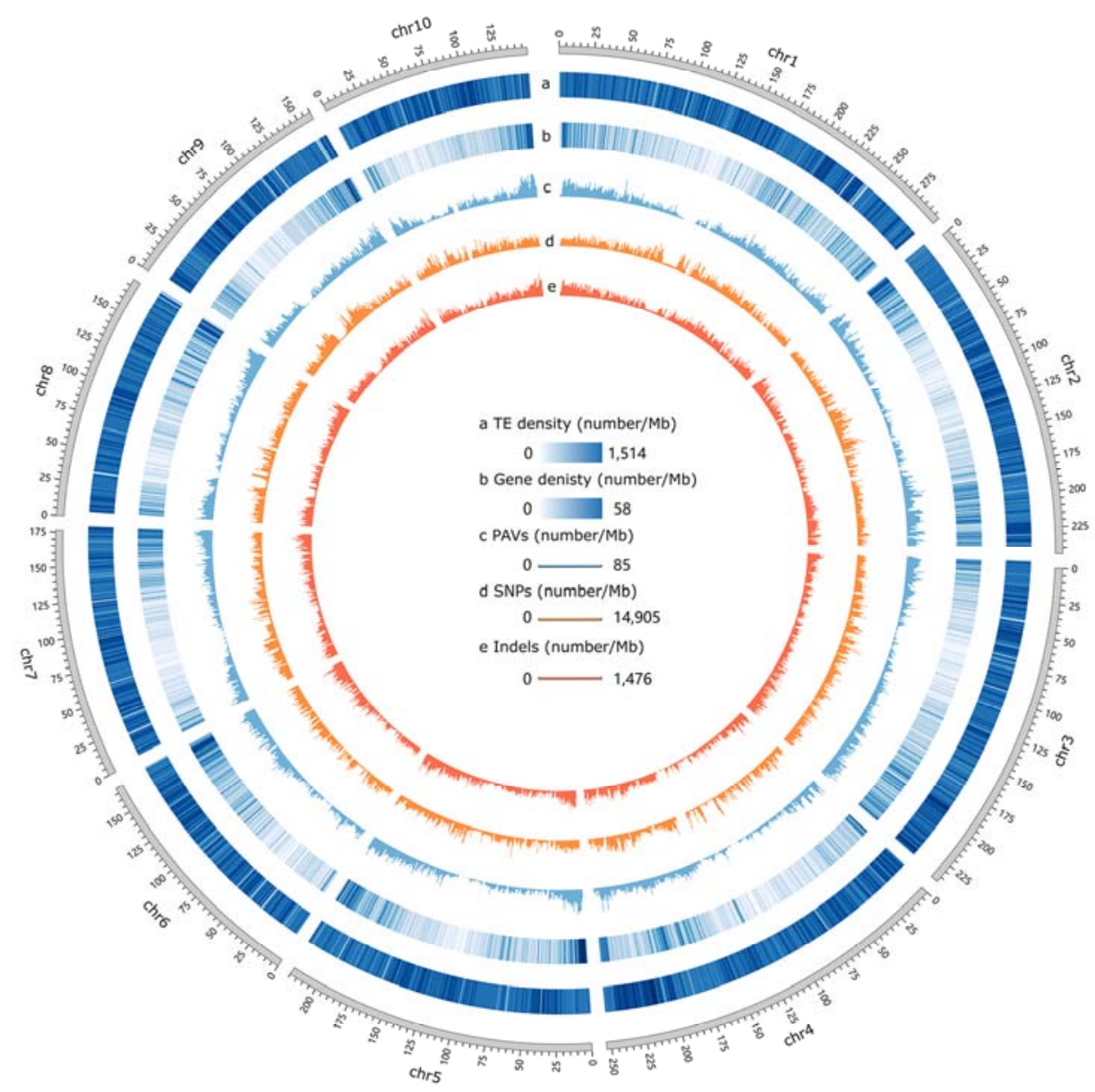

Fig. 2 Features of the A188 genome. a, Transposable-element density; b, gene density;

c, $\mathbf{d}$ and e, numbers of PAVs (c), SNPs (d) and InDels (e) compared with B73 genome.

The sliding window is $1 \mathrm{Mb}$ for all tracks. 


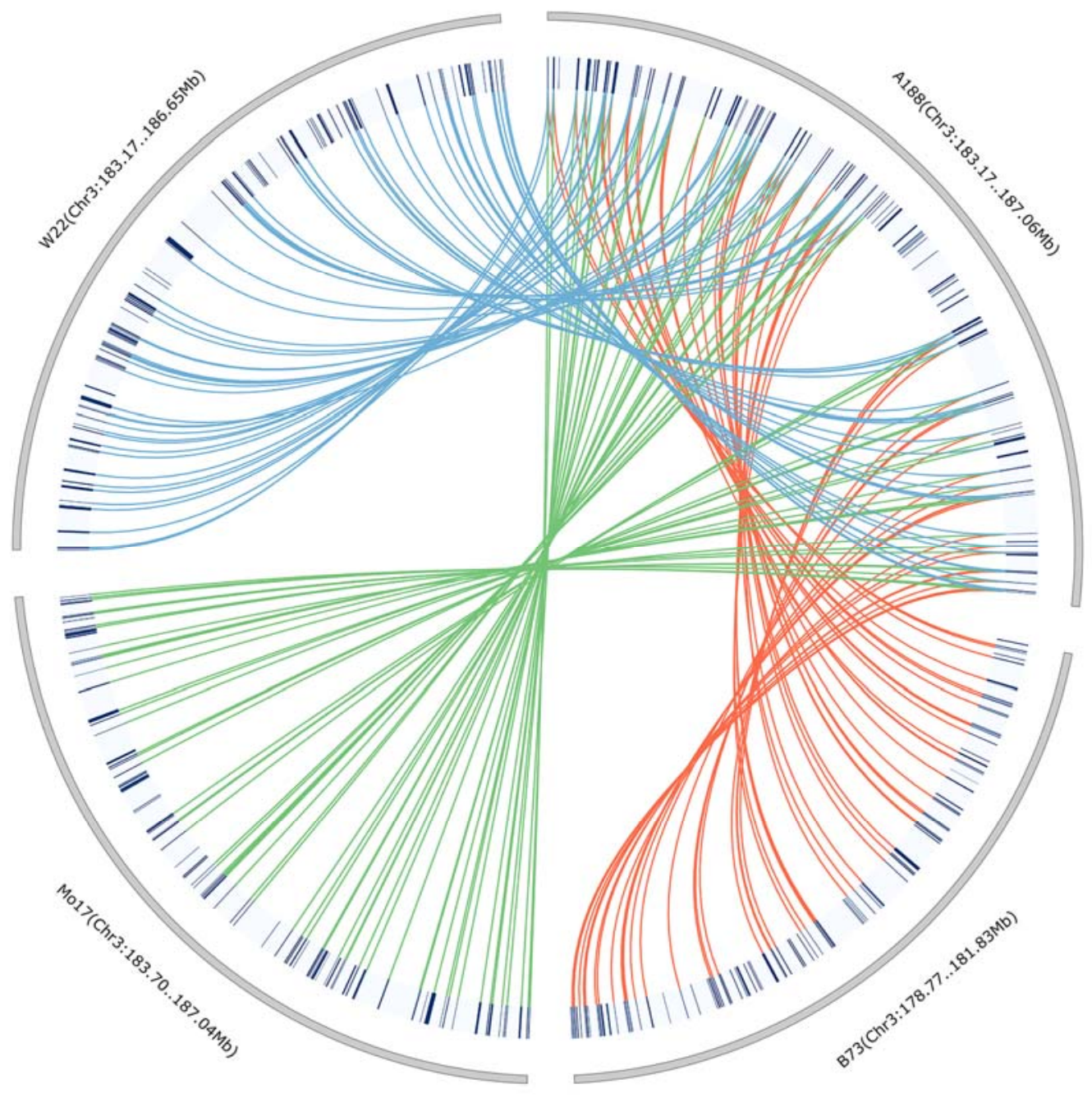

Fig. 3 Tissue culture response candidate loci. The 3.89 M A188 segment (QTL for maize tissue culture response) aligned to the syntenic segment of B73, Mo17 and W22 genomes. The red, green and blue lines indicate aligned A188 genes in the 3.89 M segment to the B73, Mo17 and W22, respectively. 


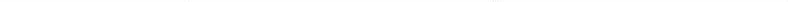

A 


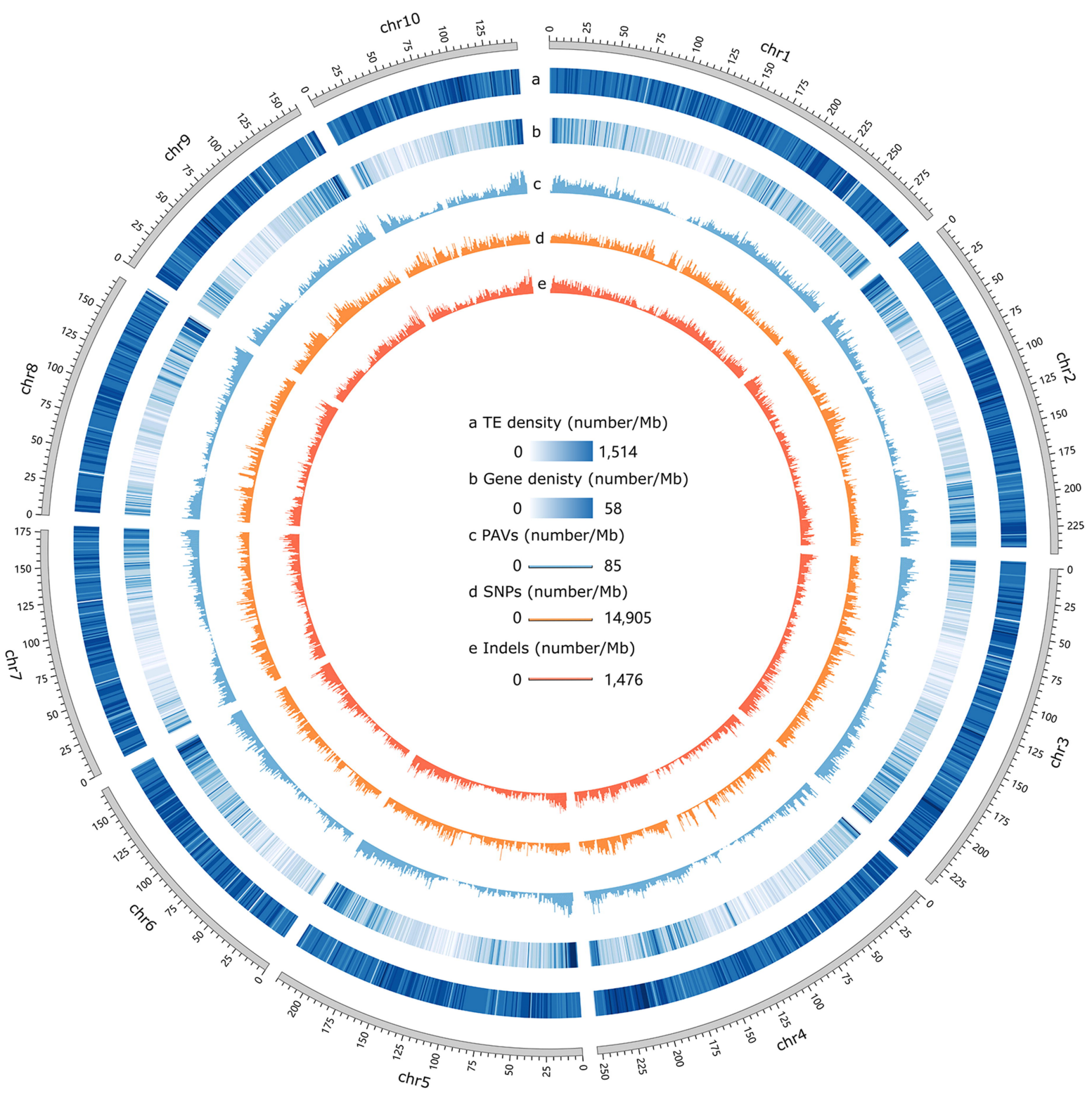




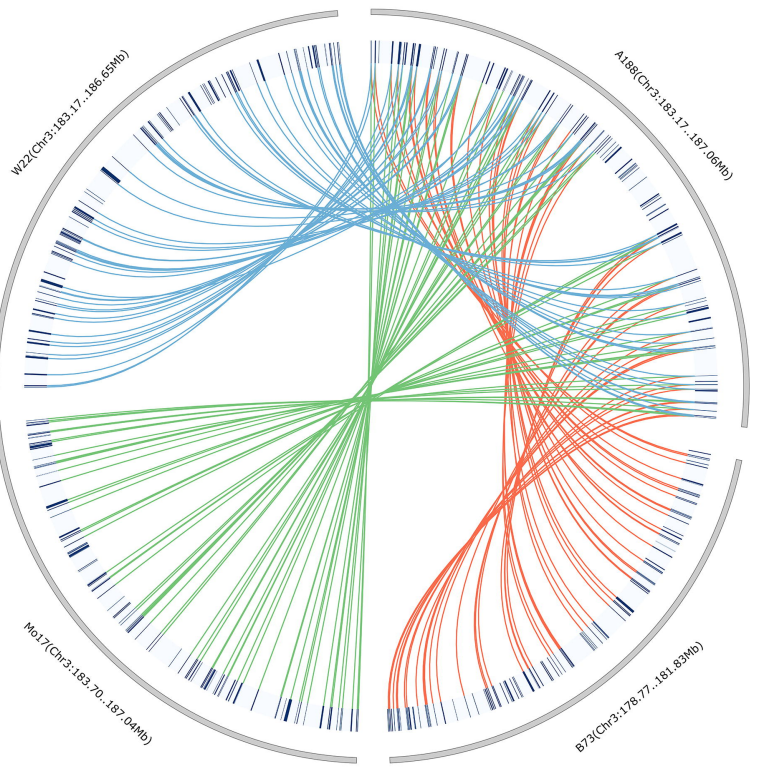

